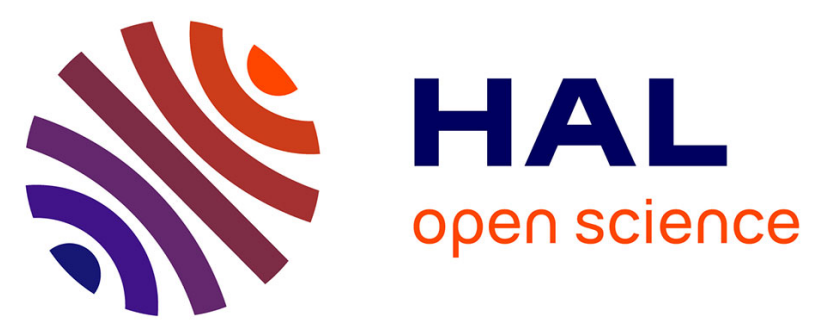

\title{
Can an electro-tactile vestibular substitution system improve balance in patients with unilateral vestibular loss under altered somatosensory conditions from the foot and ankle?
}

N. Vuillerme, P. Hlavackova, C. Franco, B. Diot, J. Demongeot, Yohan Payan

\section{To cite this version:}

N. Vuillerme, P. Hlavackova, C. Franco, B. Diot, J. Demongeot, et al.. Can an electro-tactile vestibular substitution system improve balance in patients with unilateral vestibular loss under altered somatosensory conditions from the foot and ankle?. Conference proceedings: .. Annual International Conference of the IEEE Engineering in Medicine and Biology Society. IEEE Engineering in Medicine and Biology Society. Annual Conference, 2011, 2011, pp.1323-1326. 10.1109/IEMBS.2011.6090311. hal-00666028

\section{HAL Id: hal-00666028 \\ https://hal.science/hal-00666028}

Submitted on 3 Feb 2012

HAL is a multi-disciplinary open access archive for the deposit and dissemination of scientific research documents, whether they are published or not. The documents may come from teaching and research institutions in France or abroad, or from public or private research centers.
L'archive ouverte pluridisciplinaire HAL, est destinée au dépôt et à la diffusion de documents scientifiques de niveau recherche, publiés ou non, émanant des établissements d'enseignement et de recherche français ou étrangers, des laboratoires publics ou privés. 


\title{
Can an electro-tactile vestibular substitution system improve balance in patients with unilateral vestibular loss under altered somatosensory conditions from the foot and ankle?
}

\author{
N. Vuillerme, P. Hlavackova, C. Franco, B. Diot, J. Demongeot, Y. Payan
}

\begin{abstract}
This pilot study aimed at assessing the feasibility and the effectiveness of an electro-tactile vestibular substitution system (EVSS) in patients with unilateral vestibular loss under normal and altered somatosensory conditions from the foot and ankle.

Four unilateral vestibular-defective patients voluntarily participated in the experiment. They were asked to stand upright as still as possible with their eyes closed in two Normal and Altered foot and ankle sensory conditions. In the Normal condition, the postural task was executed on a firm support surface constituted by the force platform. In the Altered condition, a $2-\mathrm{cm}$ thick foam support surface was placed under the participants' feet. These two foot and ankle sensory conditions were executed under two No EVSS and EVSS experimental conditions. The No EVSS condition served as a control condition. In the EVSS condition, participants executed the postural task using a biofeedback system whose underlying principle consisted of supplying them with additional information about their head orientation/motion with respect to gravitational vertical through electro-tactile stimulation of their tongue. Centre of foot pressure displacements (CoP) were recorded using the force platform.

Results showed that, relative to the No EVSS condition, the EVSS condition decreased CoP displacements in both the Normal and the Altered foot and ankle sensory conditions. Interestingly, the stabilizing effect was more pronounced in the Altered than in the Normal foot and ankle sensory condition.

These preliminary results suggest that patients with unilateral vestibular loss were able to take advantage to an head position-based electro-tactile tongue biofeedback to mitigate the postural perturbation induced by alteration of somatosensory input from the foot and the ankle.
\end{abstract}

\section{INTRODUCTION}

$\mathrm{T}$ he concept of "sensory substitution" that refers to the translation of sensory information that is normally available via one sense to another was pioneered by Paul Bach-y-Rita during the late 1960's [1]. Initially applied to provide distal spatial information to blind people through a tactile display [1-3], this concept has been extended to

Manuscript received April 10, 2011. This work was supported in part by the company IDS ( Montceau-les-Mines, France), the Garches Foundation, the AXA Research Fund, and the CNRS (France).

$\mathrm{N}$. Vuillerme was with the TIMC-IMAG Laboratory, UMR UJF CNRS 5525, Grenoble, France. He is now with the FRE 3405 AGIM (AGeing Imaging Modeling) Laboratory, CNRS-UJF-EPHE, Grenoble, France. (corresponding author: e-mail: Nicolas.vuillerme@agim.eu). provide a substitute body-orientation reference to individuals with vestibular dysfunction, by delivering them headposition information/motion -normally provided by the vestibular system (e.g. $[11,20])$ - via electro-tactile stimulation of the tongue [21]. Previous studies using this so called "electro-tactile vestibular substitution system" (EVSS) have reported that sensory substitution via electrotactile stimulation of the tongue was effective at improving balance control in both healthy and balance-impaired individuals [6, 7, 21-24, 27]. However, while the effectiveness of this EVSS in improving postural control during bipedal quiet standing has recently been reported in unilateral vestibular-defective patients under reliable somatosensory conditions from the foot and ankle [24], whether these patients could benefit from EVSS when subjected to challenging foot and ankle somatosensory condition remains to be investigated.

The present experiment was designed to address this issue by assessing the feasibility and the effectiveness of an electro-tactile vestibular substitution system (EVSS) in patients with unilateral vestibular loss under normal and altered somatosensory conditions from the foot and ankle.

\section{METHODS}

\section{A. Participants}

Four unilateral vestibular-defective patients voluntarily participated in the experiment. They gave their informed consent to the experimental procedure as required by the Helsinki declaration (1964) and the local Ethics Committee.

Unilateral vestibular deficit was assessed using a battery of clinical tests [24]. On the whole, a vestibular deficit was considered as unilateral if results of these tests showed an asymmetry larger than $20 \%$. To assess vestibular dysfunction, we first used a caloric test, during which bithermal caloric irrigation with cold $\left(30^{\circ} \mathrm{C}\right)$ and warm $\left(44^{\circ} \mathrm{C}\right)$ water was induced in the two ears. All patients exhibited a difference in the velocity of slow phases of nystagmus between the two ears larger than $20 \%$. To assess dynamic nonlinearities in vestibular function, we examined head-shaking nystagmus. During this test, patient shook their head vigorously about 30 times from side to side. All patients demonstrated nystagmus following head shaking. Two rotational tests under videonystagmoscopy also have 
been conducted, (1) the Rotatory Impulsion Test (RIT) at $0.05 \mathrm{~Hz}$ and (2) the High Speed Rotational Test (HSRT) at $1 \mathrm{~Hz}$. All patients exhibited a difference in number of saccades to the RIT between clockwise and counterclockwise way larger than $20 \%$. They also showed a difference in the velocity of slow phases of nystagmus to the HSRT between clockwise and counter-clockwise way larger than $20 \%$. Hearing loss averaged $43 \pm 12 \mathrm{~dB}$ in the affected ear. The history of the symptoms ranged from 6 to 10 years.

\section{B. Experimental procedure}

Participants stood barefoot on a force platform (sampling frequency: $40 \mathrm{~Hz}$ ) in a natural but standardised position (feet abducted at $30^{\circ}$, heels $3 \mathrm{~cm}$ apart), their arms hanging loosely by their sides and their eyes closed.

They were asked to stand upright as still as possible in two Normal and Altered and Improved foot and ankle sensory conditions and two conditions of No EVSS and EVSS.

In the Normal condition, the postural task was executed on a firm support surface constituted by the force platform. In the Altered condition, a 2-cm thick foam support surface was placed under the subjects' feet.

The No EVSS condition served as a control condition. In the EVSS condition, subjects executed the postural task using an electro-tactile vestibular substitution system (BrainPort Balance Device, Wicab Inc.). This system comprises two principal components: (1) the intraoral device and (2) the controller. On the one hand, the IOD is made up of an electro-tactile array, a tether, and a micro-electro mechanical system (MEMS) 3-Axis, $\pm 2 \mathrm{~g}$, digital output accelerometer. Electro-tactile stimuli are delivered to the dorsum of the tongue by the electrode array (Tongue Display Unit, TDU), which is fabricated using industry-standard photolithographic techniques for flexible circuit technology and employs a polyimide substrate. All 100 electrodes (1.5 mm diameter, on $2.32 \mathrm{~mm}$ centers) on the $24 \mathrm{~mm} \times 24 \mathrm{~mm}$ array are electroplated with a $1.5 \mu \mathrm{m}$ thick layer of gold. The tether $(12 \mathrm{~mm}$ wide $\times 2 \mathrm{~mm}$ thick) connects the electro-tactile array and accelerometer to the controller. The MEMS accelerometer, mounted on the superior surface of the electrode array, senses head position along both the anteroposterior and medioateral directions. Both the accelerometer and associated flex circuit are encapsulated in a silicone material to ensure electrical isolation for the user. On the other hand, the controller contains an embedded computer (ColdFire MCF5249C, $120 \mathrm{MHz}$, 32-bit microprocessor), stimulation circuits, user controls, and battery power supply. Custom software operating on the controller converts head-tilt signals from the accelerometer in the intraoral device into a dynamic $2 \times 2$ electrode pattern of electro-tactile stimulation. The stimulation is created by a sequence of three $25 \mu$ s wide pulses presented at a rate of $200 \mathrm{~Hz}$. The amplitude value of the pulse sequence or 'burst' is updated at $50 \mathrm{~Hz}$. Output coupling capacitors in series with each electrode assure zero net DC current to minimize the potential for tissue irritation. This waveform produces a tactile stimulus that is perceived by users as a continuous 'buzzing' or 'tingling' sensation, with minimal sensory adaptation. In the current implementation, mapping the 12-bit data to the $10 \times 10$ oral tactile array causes 'binning' of the output signal into 2.8 degree increments (both medio-lateral and anteroposterior) to individual tactor rows or columns, to a maximum range of \pm 14 degrees in each direction. Note that a pilot study with kinematic data showed that the use of a linear accelerometer alone is sufficient to provide directional information to the subject, when the device is used in the relatively static training environment. Rate sensor data coupled with linear accelerometer data could offer a more precise measure of angular and linear displacement, however, in this application, it is not necessary, as long as the stimulus displacement is in the correct direction (the direction of tilt).

Participants were asked to keep the intraoral device in their mouth all over the duration of the experiment, i.e. in both No EVSS and EVSS conditions. In the Biofeedback condition, subjects continuously perceived both position and motion of a small "target" stimulus on the tongue display, corresponding to head orientation/motion with respect to gravitational vertical. Specifically, as illustrated in Figure 1, when the participant's head sways on the left, right, forwards and backwards, the electro-tactile stimulation on the tongue moves to the left, right, forward and backward, respectively. Participants were then asked to continuously adjust their head orientation and to maintain the stimulus pattern at the centre of the display [6, 7, 21-24].

Prior to the experiment, participants performed practice trials with eyes closed on the firm and on the foam support surfaces, with and without the provision of EVSS, by voluntarily swaying in different directions. The purpose of these practice trials was for the participants to ensure that they had become familiar with standing with the foot and ankle sensory conditions and they had mastered the relationship between the different head positions and lingual electro-tactile stimulations. Data from these practice trials were not considered in the analyses.

Three 25.6-s trials for each condition were executed. The order of presentation of the two Normal and Altered foot and ankle sensory conditions and two conditions of No EVSS and EVSS was randomized. Participants were not given feedback about their postural performance. 


\section{RESULTS AND DISCUSSION}

Our initial data analysis was based on the surface area $\left(\mathrm{mm}^{2}\right)$ covered by the trajectory of the CoP, which provides a measure of the size of the CoP over the support surface.

Results showed that, relative to the No EVSS condition, the EVSS condition yielded a $29 \% \mathrm{CoP}$ surface area reduction in the Normal foot and ankle sensory condition and a $43 \% \mathrm{CoP}$ surface area reduction in the Altered foot and ankle sensory condition.

Results first confirm that patients with unilateral vestibular loss were able to efficiently take advantage of an head orientation/motion information delivered through electrotactile stimulation of the tongue to improve their postural control during quiet standing under normal foot and ankle sensory conditions [24]. This was also the case in the Altered foot and ankle sensory condition. More importantly, results further showed that the provision of the EVSS allowed them to mitigate the destabilizing effect induced by the alteration of somatosensory information at the foot and the ankle. At this point, these results extend those previously obtained in young healthy adults to a population of unilateral vestibular loss patients [23].

Although an extended study including a larger number of participants is needed to confirm these preliminary data, the larger $\mathrm{CoP}$ surface area reduction observed in the Normal than in the Altered foot and ankle sensory condition suggest that the effectiveness of the EVSS in improving postural control during bipedal standing depends on the somatosensory context. Along these lines, these results are in accordance with a previous study reporting a greater stabilizing effect provided by the EVSS observed when ankle proprioception was altered through Achilles tendon vibration than when ankle proprioception was normal [22]. Interestingly, our results obtained with vestibular-defective patients under normal and altered somatosensory from the foot and the ankle are also consistent with those recently reported in healthy subjects during vestibular disturbances induced by binaural galvanic vestibular stimulation in normal and altered support-surface conditions [27]. Indeed, EVSS has been shown to improve the control of bipedal posture during GVS toward baseline levels, again with the greatest postural improvement occurring during trials with rotation sway-referencing, i.e., in conditions of altered somatosensory conditions.

More largely, with regard to the effectiveness of a biofeedback-based intervention for improving balance control during quiet stance, the present findings are in line with previous studies reporting that the availability of a biofeedback - visual [13], vibrotactile [10, 16, 25, 26], electrotactile $[6,7,21,24]$ or auditory $[4,8,9,12]$ improves upright postural control in patients with vestibular disorders. They also support recent findings of Dozza et al. who reported that augmenting sensory information for balance control by providing audio-biofeedback related to

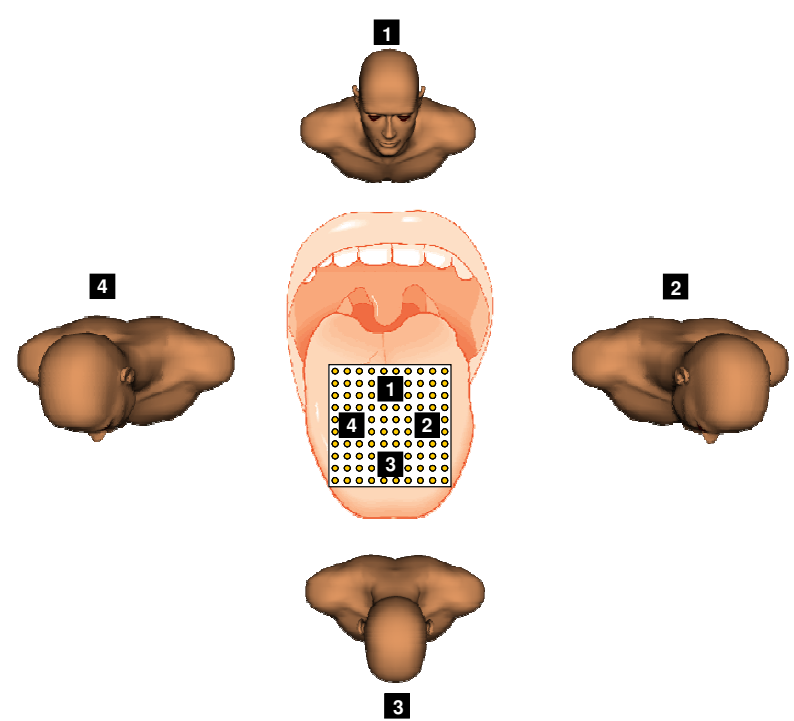

Fig. 1. Sensory coding schemes for the Tongue Display Unit as a function of the head orientation with respect to gravitational vertical: (1) extended head posture, (2) left-side-tilted posture, (3) flexed posture and (4) right-side-tilted posture

trunk acceleration to individuals with bilateral vestibular loss yielded the largest stabilizing effect when the environment provided limited somatosensory and visual information [9].

\section{ACKNOWLEDGMENT}

This work is dedicated to the memory of Professor Paul Bach-y-Rita. Paul has been for us more than a partner or a supervisor: he was a master inspiring numerous new fields of research in many domains of neurosciences, biomedical engineering and physical rehabilitation.

\section{REFERENCES}

[1] P. Bach-y-Rita, C. C. Collins, F. A Saunders, B. White, and L. Scadden, "Vision substitution by tactile image projection," Nature, vol. 221, pp. 963-964, 1969.

[2] P. Bach-y-Rita, K. A. Kaczmarek, M. E. Tyler, and J. Garcia-Lara, "Form perception with a 49-point electrotactile stimulus array on the tongue: A technical note," J. Rehabil. Res. Develop., vol. 35, pp. 427430, 1998.

[3] P. Bach-y-Rita, and S. W. Kercel, "Sensory substitution and the human-machine interface. Trends Cogn. Sci., vol. 7, pp. 541-546, 2003.

[4] D. Basta, F. Singbartl, I. Todt, A. Clarke, and A. Ernst, "Vestibular rehabilitation by auditory feedback in otolith disorders," Gait Posture, vol. 28, pp. 397-404, 2008;

[5] L. Chiari, M. Dozza, A. Cappello, F. B. Horak, V. Macellari, and D. Giansanti, "Audio-biofeedback for balance improvement: An accelerometry-based system," IEEE Trans. Biomed. Eng., vol. 52, no. 12, pp. 2108-2111, 2005.

[6] Y. P. Danilov, M. E. Tyler, K. L. Skinner, and P. Bach-y-Rita, "Efficacy of electrotactile vestibular substitution in patients with bilateral vestibular and central balance loss," Conference Proceedings-IEEE Engineering in Medicine and Biology Society, Suppl, pp. 6605-6609, 2006. 
[7] Y. Danilov, M. Tyler, K. Skinner, R. Hogle, and P. Bach-y-Rita, "Efficacy of electrotactile vestibular substitution in patients with peripheral and central vestibular loss," J. Vestib. Res., vol. 17, pp. 119-130, 2007.

[8] M. Dozza, L. Chiari, and F. B. Horak, "Audio-biofeedback improves balance in patients with bilateral vestibular loss," Arch. Phys. Med. Rehabil., vol. 86, no. 7, pp. 1401-1403, 2005.

[9] M. Dozza, F. B. Horak, and L. Chiari, "Auditory biofeedback substitutes for loss of sensory information in maintaining stance," Exp. Brain Res., vol. 178, pp. 37-48, 2007.

[10] J. A. Goebel, B. C. Sinks, B. E. Parker, N. T. Jr Richardson, A. B. Olowin, and R. W. Cholewiak, "Effectiveness of head-mounted vibrotactile stimulation in subjects with Bilateral vestibular loss : a phase 1 clinical trial," Otol. Neurotol., vol. 30, pp. 210-6, 2009.

[11] M. Green, and D. E. Angelaki, "An integrative neural network for detecting inertial motion and head orientation," J. Neurophysiol., vol. 92, pp. :905-925, 2004.

[12] J. Hegeman, F. Honegger, M. Kupper, and J. H. Allum, "The balance control of bilateral peripheral vestibular loss subjects and its improvement with auditory prosthetic feedback," J. Vestib. Res., vol. 15, no. 2, pp. 109-117, 2005.

[13] M. Hirvonen, H. Aalto, and T.P. Hirvonen, "Postural control after vestibular schwannoma resection measured with visual feedback posturography," ORL J. Otorhinolaryngol. Relat. Spec., vol. 67, pp. 335-339, 2005.

[14] F. B. Horak, and J. M. Macpherson, "Postural orientation and equilibrium". In: L. B. Rowell, and Shepard J. T., editors. "Handbook of physiology. Exercise: regulation and integration of multiple systems". Oxford: Oxford University Press; pp. 255-292, 1996.

[15] K. A. Kaczmarek, and P. Bach-y-Rita, "Tactile displays," In W. Barfield, and T. A. Furness (Eds.), Virtual environments and advanced interface design (pp. 349-414). USA: Oxford University Press, 1995.

[16] E. Kentala, J. Vivas, and C. Wall III, "Reduction of postural sway by use of a vibrotactile balance prosthesis prototype in subjects with vestibular deficits," Ann. Otol. Rhinol. Laryngol., vol. 112, pp. 404409, 2003.

[17] L. M. Nashner, F. O. Black, and C. Wall III, “Adaptation to altered support and visual conditions during stance: Patients with vestibular deficits," J Neurosci., vol. 2, pp. 536-544, 1982.

[18] R. J. Peterka, "Sensorimotor integration in human postural control," J. Neurophysiol., vol. 88, pp. 1097-1118, 2002.

[19] E. Sampaio, S. Maris, and P. Bach-y-Rita, "Brain plasticity: 'visual' acuity of blind persons via the tongue," Brain Res., vol. 908, pp. 204207, 2001.

[20] H. Schöne, "On the role of gravity in human spatial orientation," Aerospace Med, vol. 35, pp. 764-772, 1964.

[21] M. Tyler, Y. Danilov, and P. Bach-y-Rita, "Closing an open-loop control system: Vestibular substitution through the tongue," J. Integr. Neurosci., vol. 2, no. 2, pp. 159-164, 2003.

[22] N. Vuillerme, and R. Cuisinier, "Sensory supplementation through tongue electro-tactile stimulation to preserve head stabilization in space in the absence of vision," Invest. Ophthalmol. Vis. Sci., vol. 50, pp. 476-481, 2009.

[23] N. Vuillerme, N. Pinsault, O. Chenu, J. Demongeot, Y. Payan, and Y. Danilov, "Sensory supplementation system based on electrotactile tongue biofeedback of head position for balance control," Neurosci. Lett., vol. 431, pp. 206-210, 2008 a.

[24] N. Vuillerme, N. Pinsault, A. Fleury, O. Chenu, J. Demongeot, Y. Payan, and P. Pavan, "Effectiveness of an electro-tactile vestibular substitution system in improving upright postural control in unilateral vestibular-defective patients," Gait \& Posture, vol. 28, pp. 711-715, $2008 b$.

[25] C. Wall 3rd, D. M. Merfeld, S. D. Rauch, and F. O. Black, "Vestibular prostheses: The engineering and biomedical issues," J. Vestib. Res., vol. 12, no. 2-3, pp. 95-113, 2002.

[26] C. Wall 3rd, M. S. Weinberg, P. B. Schmidt, and D. E. Krebs, "Balance prosthesis based on micromechanical sensors using vibrotactile feedback of tilt," IEEE Trans. Biomed. Eng., vol. 48, pp. 1153-1161, 2001.

[27] S. J. Wood, F. O. Black, H. G. MacDougall, and S. T. Moore, "Electrotactile feedback of sway position improves postural performance during galvanic vestibular stimulation.," Ann. N. Y. Acad. Sci., vol. 1164, pp. 492-498, 2009. 